\title{
Differential phase delay observations with VLTI/MIDI at N-band
}

\author{
Robert N. Tubbs ${ }^{a}$, Jeffrey A. Meisner ${ }^{a}$, Eric J. Bakker ${ }^{a}$ and Simon Albrecht $^{a}$ \\ ${ }^{q}$ Leiden Observatory, P.O. Box 9513, 2300 RA, Leiden, Netherlands.
}

\begin{abstract}
This investigation focuses on observational measurements of the differential interferometric phase between spectral channels in the VLTI/MIDI instrument. Measurements of target stars are compared with theoretical predictions in order to investigate the effects of dispersion in humid air on differential phase measurements at $\mathrm{N}$ band ( $10 \mu \mathrm{m}$ wavelength). An accuracy of 1 degree RMS phase error is achieved after calibration during stable environmental conditions, but this accuracy is degraded if there are fluctuations in humidity between observations. Stabilisation and/or monitoring of the environmental conditions in the VLTI ducts and tunnels will be required in order to achieve the best differential phase performance with MIDI. The measured differential phases are found to be consistent with a model for the refractive index of air based on the HITRAN database.
\end{abstract}

Keywords: colour differential phase, interferometry, atmospheric turbulence, humidity, longitudinal dispersion, phase referencing, phase tracking, fringe tracking, 10 micron, MIDI, VLTI

\section{INTRODUCTION}

\subsection{Optical path length fluctuations}

Optical wavefronts passing through the Earth's atmosphere are perturbed by fluctuations in air density and water vapour density 10 Experimental measurements 5 66 indicate that the phase corrugations induced in the wavefronts change by radians on timescales of milliseconds or tens of milliseconds in the visible and near infrared, and are expected to change on timescales of tens or hundreds of milliseconds at N-band. These fluctuations inhibit direct measurements of the visibility phase for astronomical sources in mid-infrared interferometers. One of the approaches suggested for obtaining phase information for astronomical sources involves splitting the light into different spectral channels, and comparing the phases measured in each of those spectral channels. The atmospheric contributions to the phases in each of the spectral channels are expected to be correlated, allowing the differential phase between the source visibilities in each spectral channel to be extracted (see e.g. Ref. 8). Differential phase measurements can thus provide direct information about the astronomical source.

\subsection{Longitudinal dispersion at VLTI}

The two light beams from a star passing through two different VLTI telescopes will not necessarily pass through the same amount of air and water vapour en route to the MIDI beam combiner, as the (air filled) VLTI delay lines must be adjusted to compensate for the vacuum geometric delay. The variation of refractive index with wavelength in humid air causes different spectral channels to be delayed by different amounts leading to an induced differential phase delay between the

Further author information: (Send correspondence to R.N.T.)

R.N.T.: E-mail: tubbs@strw.leidenuniv.nl, Telephone: +31 715275826 


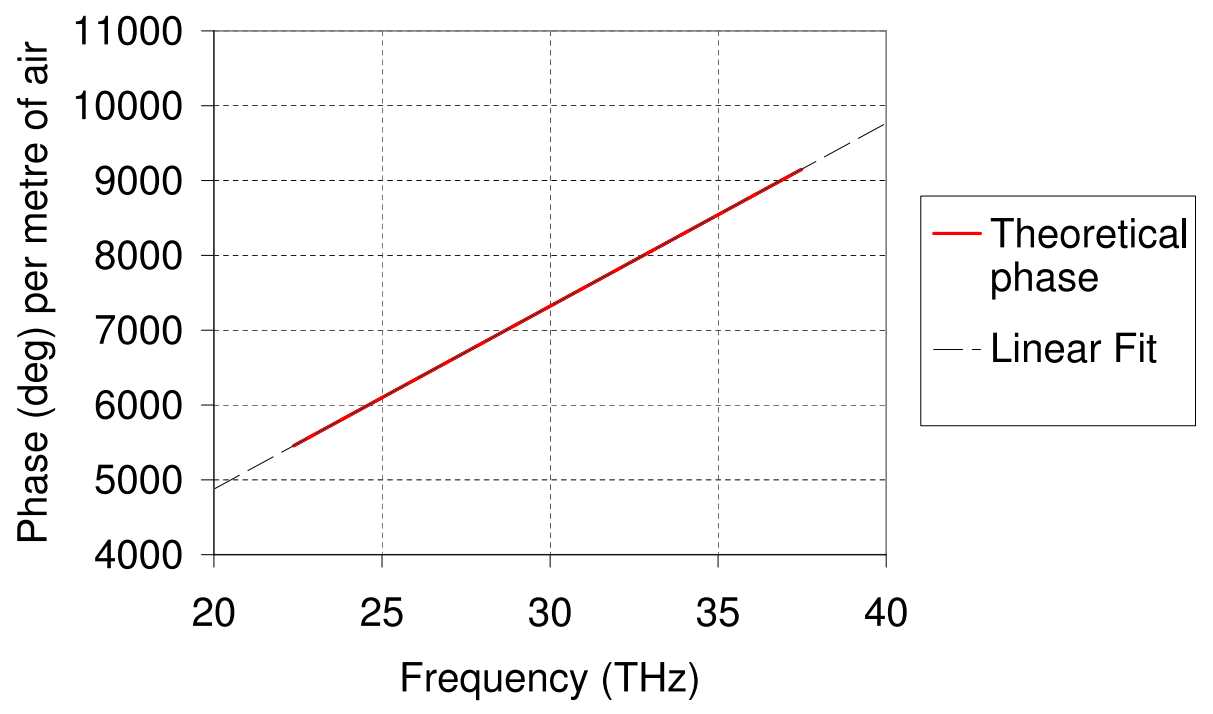

Figure 1: Theoretical prediction of phase delay induced by $1 \mathrm{~m}$ of differential air path.

spectral channels. This differential phase delay can be predicted using a model for the refractive index of air 9 The expected phase delay is labelled Theoretical Phase in Figure 1 for the case when the light in both arms of the interferometer travels the same physical distance from the star, but along one arm $1 \mathrm{~m}$ more of the path is through air. The predicted phase delay is plotted against frequency for the case of air with total molecular density (sum of all constituents) of $1.9 \times 10^{25} \mathrm{~m}^{-3}$ of which water vapour molecules contribute $4.5 \times 10^{22} \mathrm{~m}^{-3}$.

Across the $\mathrm{N}$ band the differential phase delay introduced by air refraction at relatively low humidities is well fit by a straight line as shown by the line labelled Linear Fit in Figure 1. The gradient of this line corresponds to an offset in the position of the group delay envelope (due to the mean refractive index), while the offset of the line from the origin on the phase axis corresponds to the first order contribution from longitudinal dispersion. Linear fitting performs less well at higher humidities (see e.g. Refs. 3, 9, 10), and it may be necessary to modify the fitting procedure under these conditions. 10

The turbulent fluctuations in humidity and temperature discussed in Section 1.1 also introduce fluctuations in the amount of longitudinal dispersion. The main impact of this described in terms of the theoretical plots in Figure 1 would be variations in both the gradient and the offset of the straight line fit with time (as well as smaller non-linear perturbations).

\section{THE MIDI INSTRUMENT}

The MIDI instrument at the VLTI combines beams from two telescopes with a pupil-plane beam splitter and uses temporal scanning of the fringes to measure visibilities (in a similar way to the SUSI, COAST and IOTA interferometers). For the observations presented here, pairs of $8 \mathrm{~m}$ UT apertures were used, with the UT STRAP units providing tip-tilt correction.

These observations were all performed in one of the spectrally dispersed modes of MIDI. In these modes a spectroscopic slit is placed in the image plane of the combined beams, and a pupilplane dispersing element is used to project the spectral channels across the Raytheon $320 \times 240$ IBC array detector. Observations were undertaken with two different dispersing elements: 


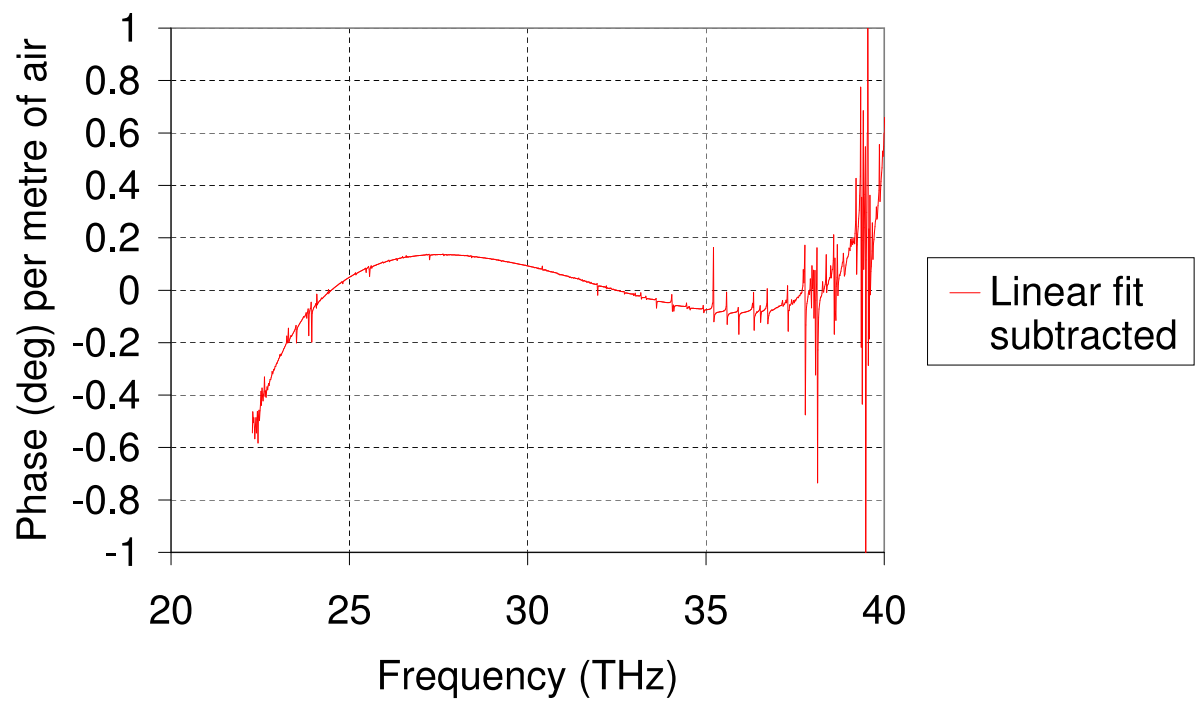

Figure 2: Theoretical prediction from Figure 1, with linear fit subtracted.

1. the MIDI PRISM, which provides approximately 25 independent spectral channels between $7.0 \mu \mathrm{m}$ and $13.4 \mu \mathrm{m}$ wavelength

2. the MIDI GRISM, which provides approximately 200 independent spectral channels between $7.0 \mu \mathrm{m}$ and $13.4 \mu \mathrm{m}$ wavelength

Both outputs of the beam combiner are recorded on the detector. The difference between the two outputs provides a good measure of the correlated flux with only a small contribution from the uncorrelated signal due to mismatch between the two beam combiner outputs.

\section{DATA REDUCTION METHOD}

\subsection{Summary}

All of the observations presented here were processed using Walter Jaffe's coherent reduction software 10 The software makes active compensation for OPD fluctuations and first-order dispersion effects by phase-rotating the measurements in each spectral channel in each atmospheric coherence time as complex numbers. For the theoretical curve of Figure 1 this would correspond to subtracting a fitted straight line from the curve, leaving only small residuals between the different spectral channels. Figure 2 shows the residuals after a fitted line was subtracted from the theoretical curve shown in Figure 1 (in a similar form to the near infrared residuals predicted by Ref. 12). I will reproduce here a brief description of the parts of Walter Jaffe's data reduction process which are relevant to differential phase observations. The reader is strongly encouraged to refer to Ref. 10 for technical details of the data reduction method.

\subsection{Simplified model of interferometric observation}

In spectrally dispersed mode the interferometric signal appears as a pattern of fringes when plotted as a function of the wavenumber $k=\frac{2 \pi}{\lambda}$ corresponding to wavelength $\lambda$. In order to explain these fringes it is useful to introduce a simplified model of an interferometric observation 
of an unresolved source which ignores longitudinal dispersion effects. In this model, the optical phase $\phi(j)$ of the $j^{\text {th }}$ spectral channel is given by the optical path difference offset $l$ from the group delay (the white-light fringe centre), expressed in terms of the wavenumber $k(j)$ and wavelength $\lambda(j)$ of the spectral channel:

$$
\phi(j)=l k(j)=\frac{2 \pi l}{\lambda(j)}
$$

In the coherent data-reduction process, the difference between the two beam combiner outputs is calculated in each spectral channel. The flux difference expected at each spectral channel for this model is given by:

$$
\begin{aligned}
I(j) & =I_{r}(j)+I_{C}(j)\left[\frac{\exp (i \phi(j))}{2}+\frac{\exp (-i \phi(j))}{2}\right] \\
& =I_{r}(j)+\frac{I_{C}(j)}{2}[\exp (i l k(j))+\exp (-i l k(j))]
\end{aligned}
$$

where $I_{r}(j)$ includes the residual uncorrelated flux at spectral channel $j, I_{C}(j)$ is the correlated flux and $i=\sqrt{-1}$.

\subsection{Group delay tracking}

For the simple model described in Section 3.2 the pattern of dispersed fringes will be given by Equation 3. The fringe pattern produced from Equation 3 for a group delay offset of $l=50 \mu \mathrm{m}$ and constant sensitivity across the observing waveband is shown in Figure 3 . The fringe spacing in this plot is proportional to the group delay offset $l$. In the MIDI coherent data reduction software the group delay offset $l$ is calculated from the fringe spacing in the spectrally dispersed measurements across $\mathrm{N}$ band using a similar approach to the group delay tracking experiment at the SUSI interferometer.13 Further details of the tracking algorithm implemented for MIDI can be found in Ref. 10 .

\subsection{Phase rotation}

The intensities in each spectral channel are "de-rotated" in the MIDI data-reduction software as complex numbers using the group delay offset $l$ measured in Section 3.3 to give a complex de-rotated intensity $I_{d}(i)$ :

$$
\begin{aligned}
I_{d}(j) & =I(j) \exp \left(-i \frac{2 \pi l}{\lambda(j)}\right) \\
& =\frac{I_{C}(j)}{2}+I_{r}(j) \exp \left(-i \frac{2 \pi l}{\lambda(j)}\right)+\frac{I_{C}(j)}{2} \exp \left(-i \frac{4 \pi l}{\lambda(j)}\right)
\end{aligned}
$$

This de-rotation process accounts for the fluctuations in optical path measured using group delay tracking (including fluctuations in the measured value of $l$ caused by e.g. the atmosphere).

The optical path difference in the MIDI instrument is artificially modulated using a piezo, leading to a modulation in $l$. This in turn causes the terms $I_{r}(j) \exp \left(-i \frac{2 \pi l}{\lambda(j)}\right)$ and $\frac{I_{C}(j)}{2} \exp \left(-i \frac{4 \pi l}{\lambda(j)}\right)$ in Equation 5 to be modulated at high frequency about zero. With the simplified model introduced in Section 3.2, averaging (or low-pass filtering) of $I_{d}(j)$ over long periods can retrieve the value of the correlated flux $I_{C}(j)$ at that spectral channel $j$.

With this simple model of an observation of an unresolved source, the phase of $I_{C}(j)$ (measured relative to the centre of the $\mathrm{N}$ band "white light fringe") will be zero. However, if the 


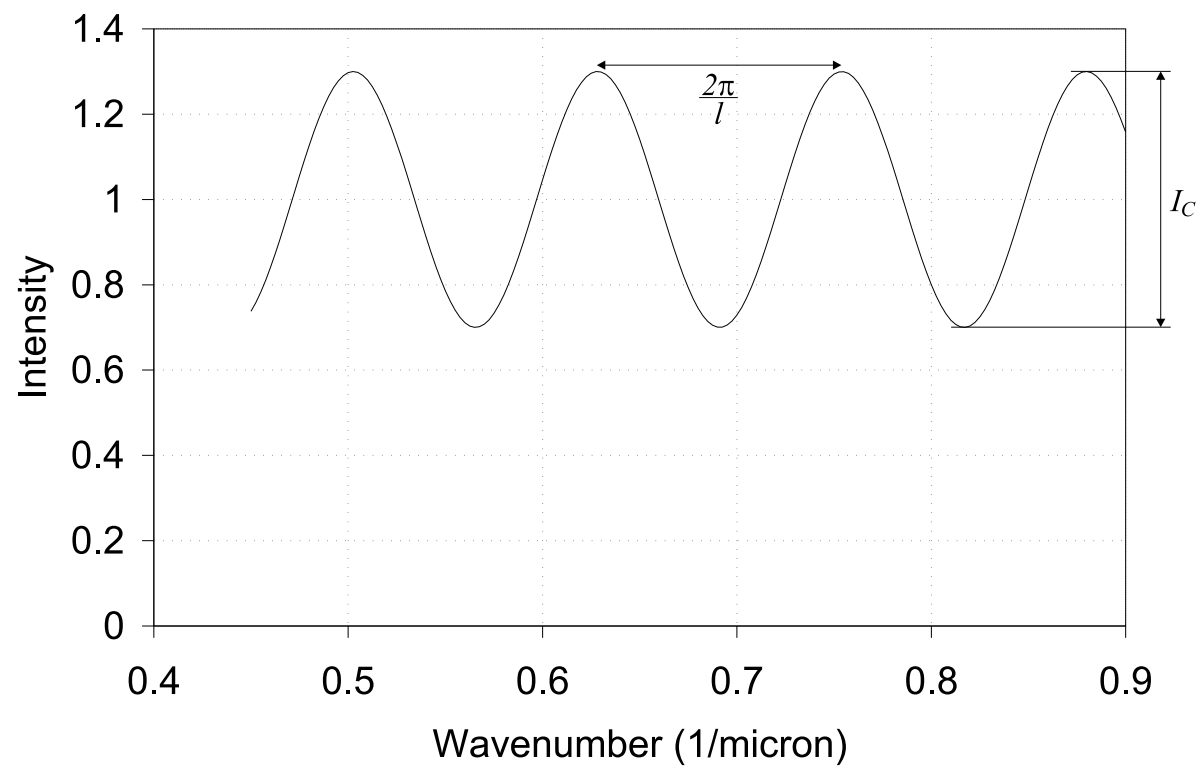

Figure 3: Fringes as a function of wavenumber in spectrally dispersed measurements from an interferometer.

source was resolved and there was an intrinsic phase difference between two or more of the spectral channels, the phase delay between the spectral channels would be preserved in the data reduction process. Note that as phases are all measured relative to the N-band mean group delay, the absolute phase of the spectral channels is lost and only partial information about the differential phases between spectral channels is preserved.

In an additional stage of the MIDI coherent data reduction software, the correlated fluxes in all the spectral channel are summed:

$$
I_{S}=\sum I_{C}(j)
$$

The phase of this sum is subtracted from the phases for the individual spectral channels (the phase of $I_{d}(j)$ is de-rotated by the same amount for each channel $j$ ). In terms of the phases in the theoretical curve shown in Figure 1 this corresponds to subtracting the offset of a fitted straight line. When combined with correction of the group delay offset, this only leaves small non-linear residuals (Figure 2). This process removes the effects of longitudinal dispersion to first order and is repeated at each atmospheric coherence time. When combined with the group delay correction it allows coherent addition of visibility data from many atmospheric coherence times (as discussed in Ref. 10).

\section{OBSERVATIONS}

Only spectrally dispersed data from MIDI can be used for making differential phase measurements. In this paper I will primarily discuss MIDI PRISM mode observations taken on the night of 2003 June 12, although I will include a brief discussion of some data from 2003 June 14 PRISM observations, and a MIDI GRISM mode observation from 2004 February 2. 
Table 1: Targets on night of 2003 June 12

\begin{tabular}{|l|l|l|}
\hline Run & Target Name & $\begin{array}{l}\text { Time on June 13 } \\
\text { (UT hh:mm:ss) }\end{array}$ \\
\hline 1 & HD 89388 & $00: 01: 10$ \\
\hline 2 & HD 89388 & $01: 05: 09$ \\
\hline 3 & HD 125687 & $01: 54: 59$ \\
\hline 4 & HD 120323 & $04: 28: 33$ \\
\hline 5 & HD 120323 & $05: 26: 50$ \\
\hline 6 & HD 168454 & $05: 53: 41$ \\
\hline 7 & HD 168454 & $08: 19: 14$ \\
\hline 8 & HD 168454 & $10: 37: 07$ \\
\hline
\end{tabular}

\subsection{Data taken on 2003 June 12}

2003 June 12 was unusual in that the relative humidity at the Paranal weather station was very uniform throughout the night (remaining at $11 \% \pm 1 \%$ ). The mean water vapour and air density at the weather station were taken to represent a good estimate for the environmental conditions within the VLTI. All the interferometric data were taken using the UT1-UT3 baseline. Spectrally dispersed observations of targets which were not strongly resolved by this baseline (mostly MIDI calibrator stars) were chosen. The observations used in are listed in Table 1 .

\subsection{Data taken on 2003 June 14}

On the night of 2003 June 14 the relative humidity showed more extreme fluctuations, and the relative humidities measured at the weather station are not expected to accurately reflect the conditions within the VLTI. Data from June 14 are only included here to demonstrate that accurate differential phases cannot be calculated if the humidity in the VLTI is not stable or not measured.

\subsection{Data taken on 2004 February 2}

On 2004 February 2 the relative humidity also showed substantial fluctuations and astronomical seeing conditions were relatively poor, limiting the interferometric data quality which could be obtained on this night. One observation using MIDI GRISM mode from 2004 February 2 is included to demonstrate the potential of differential phase measurements using the MIDI GRISM.

\section{RESULTS}

Data from the eight runs (calibration observations) listed in Table 1 were processed in a semiautomated fashion using Walter Jaffe's coherent data reduction software 10 The interferometric phase was measured with good signal-to-noise in all the MIDI spectral channels between $7.8 \mu \mathrm{m}$ and $13.4 \mu \mathrm{m}$ on all the targets. The highest signal-to-noise was generally found in two regions of the spectrum: between $8.5 \mu \mathrm{m}$ and $9.3 \mu \mathrm{m}$; and between $10 \mu \mathrm{m}$ and $13 \mu \mathrm{m}$. As discussed in Section 3 and Ref. 10, the data reduction method adjusts the first and second order Taylor expansion components of differential phase as a function of frequency. These components were subsequently set to zero in this analysis (equivalent to subtracting the best-fit line in Figure 1), 


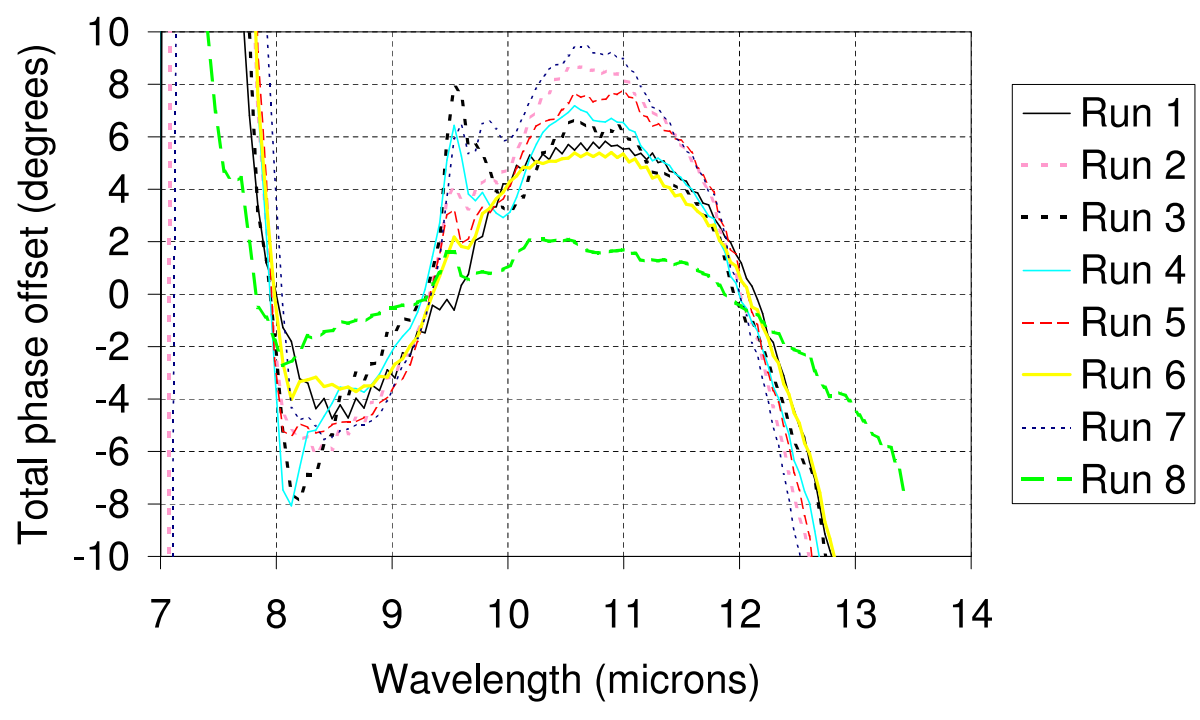

Figure 4: Phase offset as a function of wavelength for PRISM observations taken on 2003 June 12.

and the resulting data has been plotted in Figure 4 for the eight runs. The residual (higher order) components shown in Figure 4 have similar shapes for each of eight observations, although the magnitudes differ. The largest differences in shape appear near to $9.6 \mu \mathrm{m}$ wavelength, around the N-band atmospheric ozone absorption lines.

In order to find out whether the different magnitudes for the residuals in Figure 4 are simply a result of the varying differential air paths in the VLTI (up to $80 \mathrm{~m}$ in these observations), each of the curves was divided by the mean differential air path for that observation. The resulting curves provide a measure of the phase offset per metre of air path, and are shown in Figure 5 . Also shown in the figure is the theoretical residual component predicted by the model of Richard Mathar $\frac{9}{9}$ after the first and second order components of differential phase as a function of frequency had been set to zero (not to be confused with first and second order dispersion terms). The theoretical plot was calculated using air and water vapour densities consistent with the mean conditions measured at the Paranal weather station on this observing night. There is excellent agreement between all the curves apart from a small region around the N-band ozone absorption lines. The first run of the night (Run 1) shows slightly larger deviations, which might be because the air within the VLTI delay line had not reached equilibrium with the outside air when the observation was performed (although there is no other evidence which can confirm this). The large deviations for Run 8 in this plot may be a result of the smaller differential air path for this observation.

The differential phase measurements shown in Figure 5 are generally consistent with the effects of longitudinal dispersion in air, and there is no evidence here for systematic differential phase contributions from the VLTI optics. The deviations around $9.6 \mu \mathrm{m}$ wavelength might be the result of fluctuations in the density of ozone within the VLTI, or due to another gas species with absorption lines in a similar part of the spectrum, although there is no other evidence to support either of these hypotheses. The interferometric signal-to-noise is generally slightly lower around $9.6 \mu \mathrm{m}$ wavelength due to ozone absorption lines in the upper atmosphere, and it is possible that this might be the cause of the increased differential phase errors in this part of 


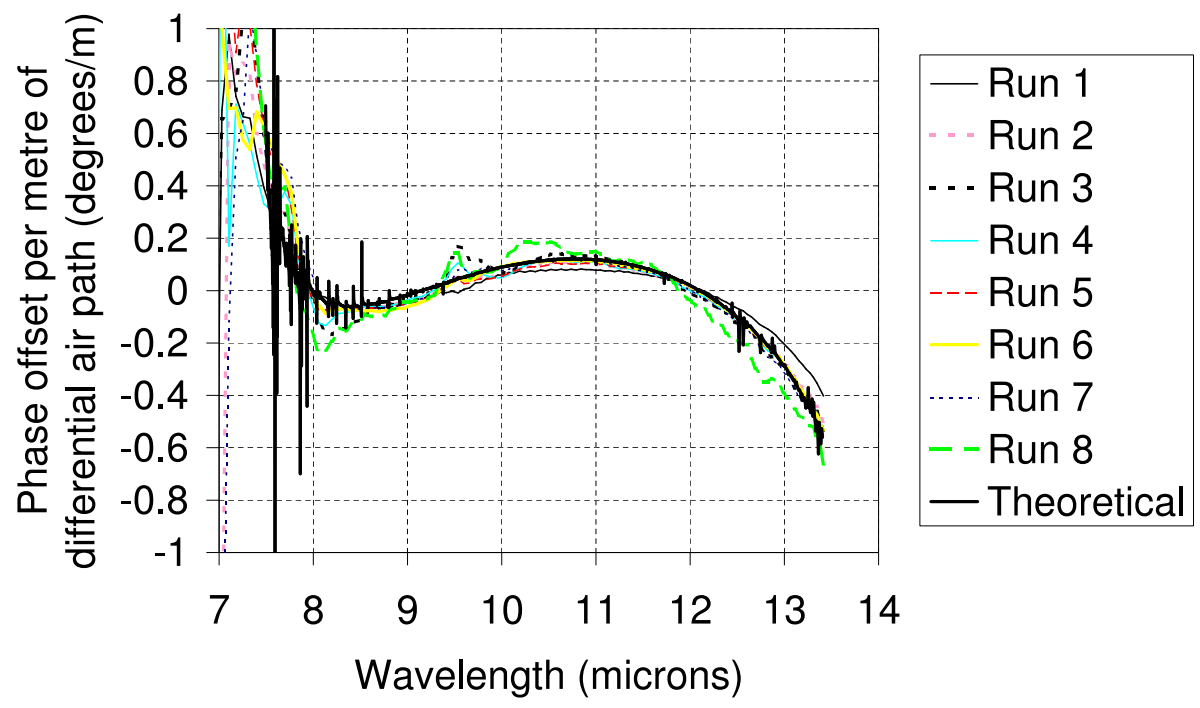

Figure 5: Phase offset per metre of differential air path for the data in Figure 4 . A theoretical prediction based on environmental measurements at the Paranal weather station and on a refractive index model from Ref. 9 is also shown.

the spectrum. Fluctuations in the ozone density in the atmosphere could also introduce phase fluctuations in this part of the spectrum.

If differential phase observations were applied to astronomical sources, the measurements on a target object would be calibrated using observations of well-understood calibrator stars taken immediately before and/or after the target observation. The calibrator star phase observations would then be scaled in order to account for the change in differential air path in the VLTI between the source and calibrator observations. In order to provide a visual indication of the agreement between the measurements on June 12, the mean of all the measured data in Figure 5 was taken. This was then used to calibrate each of the individual measurements by scaling it and subtracting it from each of the curves in Figure 4 in turn to leave the residuals plotted in Figure 6. The dataset is large enough that the correlated errors are fairly negligible, so that this plot gives a good idea of the accuracy which can be achieved when using an average calibration based on the data from a whole night (albeit one with relatively stable humidity). If the environmental conditions vary during the night it is better to only use calibrator observations taken at times close to the science target observation. Pairs of concurrent measurements were selected from those used for Figure 4. The first measurement in each pair was scaled according to the ratio of the differential air paths so as to act as a calibration for the second measurement. The differences between the measurements were then computed, and were found to be typically less than 1 degree RMS over the parts of the spectrum with high signal-to-noise. In science observations (on resolved objects), the differential phases may change by up to 180 degrees, and the measurement accuracy demonstrated by Figure 6 should strongly constrain the source geometries. Preliminary results from resolved sources show a phase change of $\sim 180$ degrees across a visibility null in the spectrum of one source as would be expected for a symmetric source.

There were larger fluctuations in humidity on 2003 June 14 and much larger variation was seen in the differential phases for calibrator stars. A similar calibration procedure to that used 


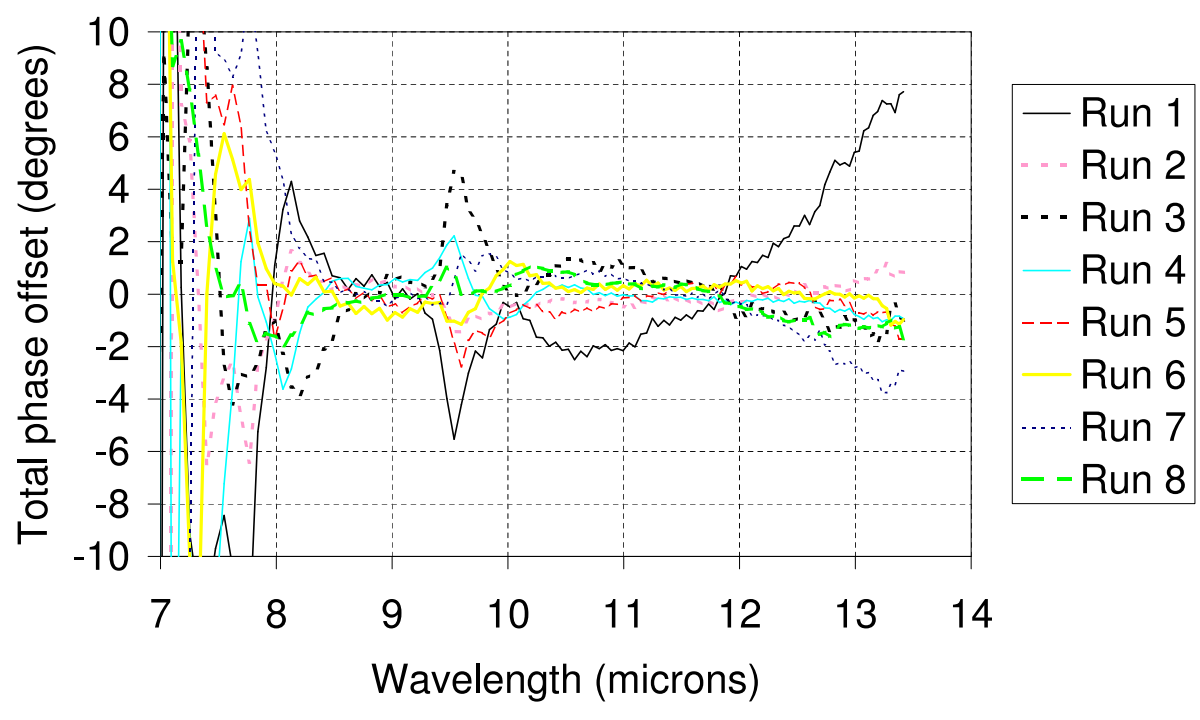

Figure 6: The observations from Figure 4 after calibration for longitudinal dispersion in the atmosphere. The calibration procedure provides a substantial reduction in the scatter of differential phases for these calibration stars.

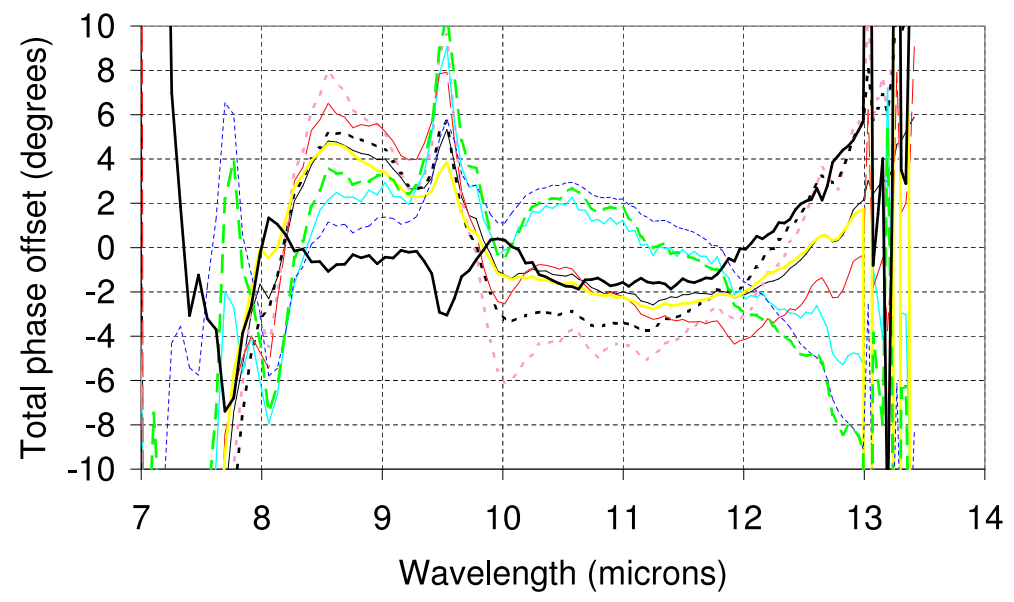

Figure 7: PRISM observations from 2003 June 14 calibrated in a similar way to the data in Figure 6

for Figure 6 was applied to the June 14 data, and the remaining phase errors were about three times larger, as can be seen in Figure 7.

In future many MIDI observations will be performed in GRISM mode with a spectral resolution of $\sim 300$. In order to ensure that GRISM mode data is suitable for differential phase observations, the first calibrator observation from 2004 February 2 has been analysed here. The environmental conditions were generally quite poor in early February with frequent changes in the humidity, but the humidity remained relatively stable early in this night. The measured differential phases agreed well with predictions based on the environmental conditions at the 


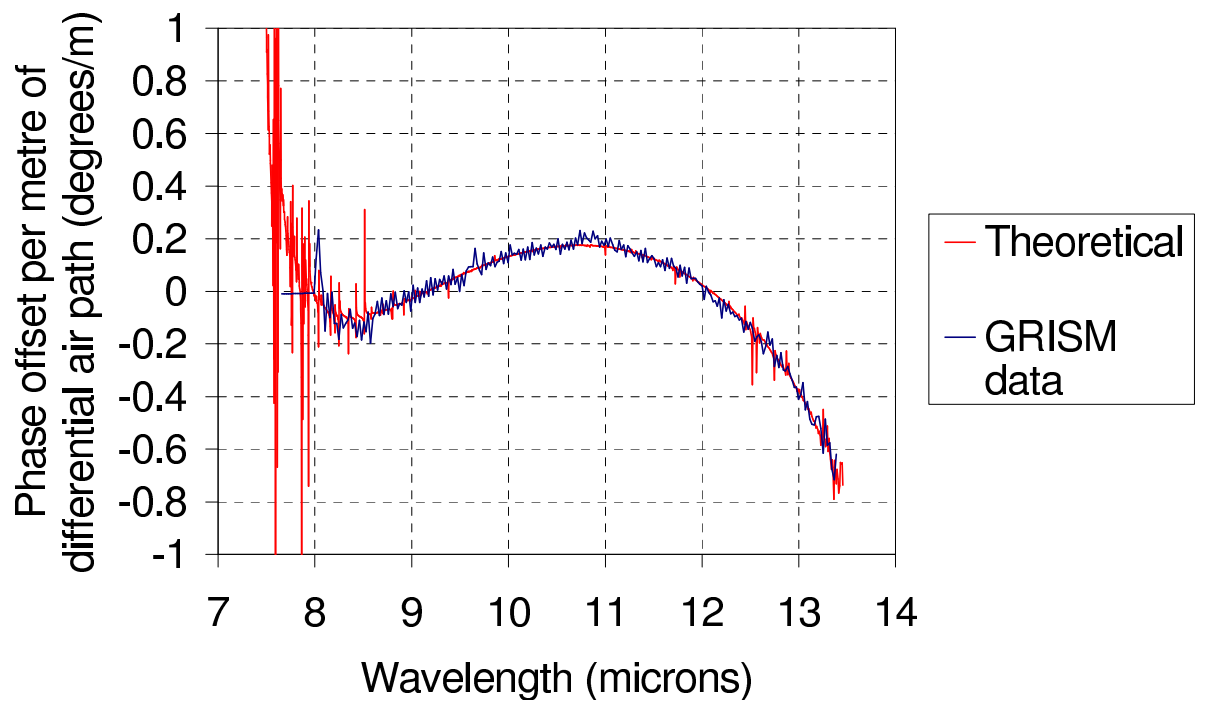

Figure 8: Phase measured per metre of air path in a GRISM observation on 2004 February 2 compared with a theoretical prediction based upon Ref. 9

Paranal weather station, as can be seen in Figure 8. Fluctuations in the ambient humidity later in the night resulted in poorer agreement for later observations.

\section{DISCUSSION}

Differential phase measurements may provide stronger constraints on astronomical models than the visibility amplitudes provided by MIDI due to the relatively high precision of these measurements. They are particularly well-suited for the detection of visibility nulls (which result in a $\sim 180$ degree phase change) and for detecting source asymmetry. Multi-wavelength models of the astronomical source are required for model fitting, however.

In order to regularly obtain high-precision differential phase measurements, it may be necessary to monitor the humidity, temperature and pressure in the VLTI ducts and delay line tunnel during observations. A set of four humidity and temperature sensors has already been calibrated in Leiden ${ }^{14}$ for this purpose. These sensors will be arranged in the VLTI ducts and the main delay line in order to assess the impact of water vapour on MIDI and GENIE observations at the VLTI.

Further improvement in the accuracy of differential phase measurements might be attained by sealing off the VLTI ducts and tunnels from fluctuations in the outside environment using (exchangeable) windows at the telescopes. Finding window materials which would work at both $1.7 \mu \mathrm{m}$ and $10 \mu \mathrm{m}$ might be difficult however, preventing joint FINITO/MIDI observations under these conditions.

The data reduction software currently fits longitudinal dispersion to first order by adjusting the mean phase across N-band. One improvement to the software suggested in Ref. 10 would be to fit realistic models for the longitudinal dispersion of air and water vapour. The software is not optimised for observations of sources with strong differential phase signatures (although it has been found to work successfully on bright targets with differential phase changes of up to 
180 degrees). An iterative method ${ }^{10}$ could be used to provide better-optimised results on highly resolved or asymmetric targets.

\section{CONCLUSIONS}

Differential phase delay accuracies of 1 degree are possible after subtracting the mean phase and the gradient of the phase with spectral frequency. This will be extremely useful for many of the MIDI science projects. Differential phase measurements may provide additional constraints on astronomical models that are not provided by visibility amplitudes measurements with MIDI. In order to obtain high accuracy differential phases regularly, monitoring 14 and/or stabilisation of the environment in the VLTI may be required. It is hoped that improvements to the software suggested in Ref. 10] will further enhance the differential phase capabilities of MIDI.

\section{ACKNOWLEDGMENTS}

This work has relied on software ${ }^{10}$ developed at Leiden Observatory and on observational data made available through the ESO/MIDI Consortium Guaranteed Time Observing program.

\section{REFERENCES}

1. F. Roddier, "The effects of atmospheric turbulence in optical astronomy," in Progress in optics. Volume 19. Amsterdam, North-Holland Publishing Co., pp. 281-376, 1981.

2. D. Saint-Jacques, Astronomical Seeing in Space and Time - available at http://olbin.jpl.nasa.gov/theses/dsj-thesis.ps. PhD thesis, Cambridge University, 1998.

3. J. A. Meisner and R. S. Le Poole, "Dispersion affecting the VLTI and 10 micron interferometry using MIDI," in Proc. SPIE, Vol. 4838, Interferometry for Optical Astronomy II, W. Traub Ed., pp. 609-624, Feb. 2003.

4. D. F. Buscher, J. T. Armstrong, C. A. Hummel, A. Quirrenbach, D. Mozurkewich, K. J. Johnston, C. S. Denison, M. M. Colavita, and M. Shao, 'Interferometric seeing measurements on Mt. Wilson: power spectra and outer scales," Applied Optics 34, pp. 1081-1096, Feb. 1995.

5. N. S. Nightingale and D. F. Buscher, 'Interferometric seeing measurements at the La Palma Observatory," Monthly Notices of the Royal Astronomical Society 251, pp. 155-166, July 1991.

6. J. W. O'Byrne, "Seeing measurements using a shearing interferometer," Publications of the Astronomical Society of the Pacific 100, pp. 1169-1177, Sept. 1988.

7. M. M. Colavita, M. Shao, and D. H. Staelin, 'Atmospheric phase measurements with the Mark III stellar interferometer," Applied Optics 26, pp. 4106-4112, Oct. 1987.

8. R. L. Akeson, M. R. Swain, and M. M. Colavita, 'Differential phase technique with the Keck Interferometer," in Proc. SPIE, Vol. 4006, Interferometry in Optical Astronomy, P. Lena, A. Quirrenbach Eds., pp. 321-327, July 2000.

9. R. J. Mathar, "Calculated Refractivity of Water Vapor and Moist Air in the Atmospheric Window at $10 \mu \mathrm{m}$," Applied Optics 43, pp. 928-932, Feb. 2004.

10. W. Jaffe, "Coherent Fringe Tracking and Visibility Estimation for MIDI," in Proc. SPIE, Vol. 5491, New Frontiers in Stellar Interferometry, W. Traub Ed., p. (these proceedings), June 2004.

11. W. Jaffe, "For further information see the vlti/midi data reduction, analysis and science school, leiden university observatory http://www.strw.leidenuniv.nl/ nevec/workshop_2004/index.html," Oct. 2004. 
12. G. Daigne and J.-F. Lestrade, 'Astrometric optical interferometry with non-evacuated delay lines," Astronomy and Astrophysics Supplement Series 138, pp. 355-363, Aug. 1999.

13. P. R. Lawson, "Group-delay tracking in optical stellar interferometry with the fast Fourier transform," J. Opt. Soc. Am. A, vol. 12, page 366 received September 2 12, pp. 366+, 1995.

14. S. Albrecht, E. J. Bakker, J. A. de Jong, R. N. Tubbs, J. A. Meisner, and R. S. le Poole, "Calibration of temperature and relative humidity sensors for use on the VLT-Interferometer," in Proc. SPIE, Vol. 5491, New Frontiers in Stellar Interferometry, W. Traub Ed., p. (these proceedings), June 2004. 